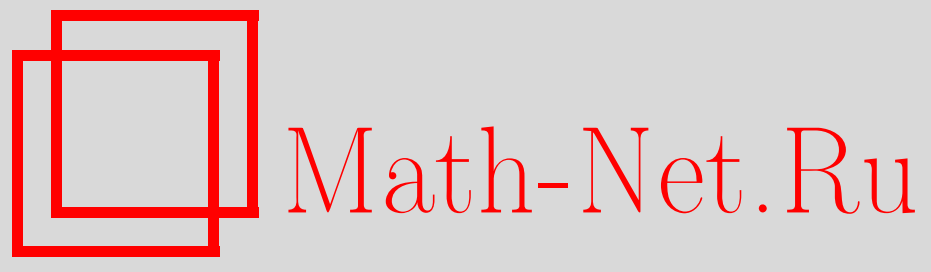

О. П. Филатов, Теорема об усреднении для неопределенных условно-периодических движений, Матем. заметки, 2011, том 90, выпуск 2, 318-320

DOI: https://doi.org/10.4213/mzm8886

Использование Общероссийского математического портала Math-Net.Ru подразумевает, что вы прочитали и согласны с пользовательским соглашением http://www . mathnet.ru/rus/agreement

Параметры загрузки:

IP : 52.205 .19 .152

26 апреля 2023 г., 14:24:56

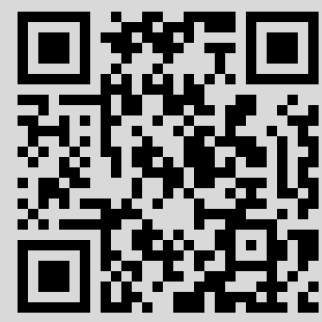




\section{Теорема об усреднении для неопределенных условно-периодических движений}

\section{О. П. Филатов}

Рассмотрим интегрируемую по Риману функцию $f(y)$ на $n$-мерном торе $\mathbb{T}^{n}$, где $y=$ $\left(y_{1}, \ldots, y_{n}\right) \bmod 2 \pi-$ угловые координаты. Зафиксируем вектор $\omega=\left(\omega_{1}, \ldots, \omega_{n}\right)$, число $\alpha \in(0,1]$ и начальный вектор $a=\left(a_{1}, \ldots, a_{n}\right)$. Дифференциальное включение

$$
\dot{y} \in\{\alpha \omega, \omega\}, \quad y(0)=a,
$$

определяется двумя допустимыми скоростями $\alpha \omega$ и $\omega$. Решением задачи (1) называется функция $y:[0, \infty) \rightarrow \mathbb{R}^{n}, y(0)=a$ абсолютно непрерывная на любом отрезке из промежутка $\mathbb{R}_{+}=[0, \infty)$, производная которой $\dot{y}$ почти всюду принадлежит множеству допустимых скоростей. Если $\alpha=1$, то единственное решение $y(t)=a+t \omega$ задачи (1) задает условно-периодическое движение на торе. Если координаты вектора $\omega$, называемые частотами, независимы (равенство $k_{1} \omega_{1}+\cdots k_{n} \omega_{n}=0$ для целочисленных $k_{1}, \ldots, k_{n}$ влечет $\left.k_{1}=\cdots=k_{n}=0\right)$, функция $f(a+t \omega)$ локально интегрируема по Риману в промежутке $\mathbb{R}_{+}$, то пространственное среднее функции $f$ на торе $\mathbb{T}^{n}$, определяемое формулой

$$
m(f)=\frac{1}{(2 \pi)^{n}} \int_{K} f(y) d y, \quad K=[0,2 \pi] \times \cdots \times[0,2 \pi],
$$

совпадает с временным средним

$$
\lim _{\tau \rightarrow \infty} \frac{1}{\tau} \int_{0}^{\tau} f(a+t \omega) d t
$$

Этот результат установлен в 1909 г. и известен как теорема об усреднении для систем, совершающих условно-периодическое движение [1]. В данной работе устанавливается связь пространственного среднего $m(f)$ и предела максимального среднего

$$
M(f)=\lim _{\tau \rightarrow \infty} \sup _{y \in Y} \frac{1}{\tau} \int_{0}^{\tau} f(y(t)) d t,
$$

где $Y$ - множество всех решений задачи (1).

Пусть $\lambda(\cdot), \mu(\cdot)$ - соответственно меры Жордана на $K$ и $\mathbb{R}, f_{0}=f-m(f)$,

$$
K_{c}=\left\{y \in K: f_{0}(y) \geqslant c\right\}, \quad I_{c}=\int_{K_{c}} f_{0}(y) d y, \quad \lambda_{c}=\lambda\left(K_{c}\right) .
$$

Кроме того, обозначим $k=1 / \alpha$ и введем функцию

$$
\varphi(k, c)=\frac{(k-1) I_{c}}{(2 \pi)^{n}+(k-1) \lambda_{c}} .
$$

Стандартным способом разобьем $n$-мерный куб $K$ на попарно непересекающиеся кубики $K_{j}, j \in J$, число которых $|J|=l^{n}, l=1,2, \ldots$. Множество индексов $j \in J$, которое отвечает включению $K_{j} \subset K_{c}$ (пересечению $K_{j} \cap K_{c} \neq \varnothing$ ) обозначим символом $J_{c, 1}\left(J_{c, 2}\right)$. Введем элементарные аппроксимирующие множества

$$
K_{c, 1}=\bigcup_{j \in J_{c, 1}} K_{j}, \quad K_{c, 2}=\bigcup_{j \in J_{c, 2}} K_{j}
$$

для жорданова множества $K_{c}$. 
Семейство множеств $\left\{K_{c}\right\}, c \in \mathbb{R}_{+}$, называется равномерно измеримым, если для любого $\varepsilon>0$ существует такое $l_{0}$, что для любого целого $l \geqslant l_{0}$ и любого $c \in \mathbb{R}_{+}$выполняется неравенство $\lambda\left(K_{c, 2}\right)-\lambda\left(K_{c, 1}\right) \leqslant \varepsilon$.

Например, если граница любого множества $K_{c}$ имеет $(n-1)$-мерную жорданову меру (при $n=2$ - это длина спрямляемой кривой), которая не превосходит некоторой общей для всего семейства постоянной, то такое семейство удовлетворяет условию равномерной измеримости. Для $\tau>0$ введем обозначения

$$
\begin{gathered}
R_{c, \tau}=\left\{t \in[0, \tau]:(a+t \omega) \in K_{c}\right\}, \quad R_{j, \tau}=\left\{t \in[0, \tau]:(a+t \omega) \in K_{j}\right\}, \\
R_{j, \tau, c}=\left\{t \in[0, \tau]:(a+t \omega) \in K_{j} \cap K_{c}\right\}, \\
r_{1}=\sup _{y \in K}\left|f_{0}(y)\right|, \quad r=\max \left\{1, r_{1}\right\} .
\end{gathered}
$$

Теорема 1. Пусть частоты $\omega_{1}, \ldots, \omega_{n}$ независимы, функиия $f$ интегрируема на $\mathbb{T}^{n}$ по Риману, а $f(a+t \omega)$ локально интегрируема на $\mathbb{R}_{+}$по Риману и при любых $c \in \mathbb{R}$, $\tau>0$ множества $K_{c}, R_{c, \tau}$ измеримы по Жордану, а семейство множеств $\left\{K_{c}\right\}, c \in \mathbb{R}_{+}$, равномерно измеримо. Тогда предел максимального среднего $M_{f}$ вычисляется по формуле

$$
M(f)=m(f)+\sup _{c \geqslant 0} \varphi(k, c) .
$$

ДокАЗАТЕльство. В силу теоремы усреднения [1; с. 251-254] пространственное среднее функции $f_{0}$, равное 0 , совпадает с временным средним функции $f_{0}(a+t \omega)$. Следовательно, имеет место соотношение [2; теорема 1$]$

$$
M\left(f_{0}\right)=\lim _{\tau \rightarrow \infty} \sup _{c \geqslant 0} \frac{(k-1) I_{c, \tau}}{1+(k-1) \mu_{c, \tau}},
$$

которое было доказано в работе [2] в более общем случае интегрируемой по Лебегу функции $f_{0}: \mathbb{R} \rightarrow \mathbb{R}$. Здесь

$$
I_{c, \tau}=\frac{1}{\tau} \int_{R_{c, \tau}} f_{0}(a+t \omega) d t, \quad \mu_{c, \tau}=\frac{\mu\left(R_{c, \tau}\right)}{\tau} .
$$

Пусть $\chi_{c}$ характеристическая функция множества $K_{c}$. Точную нижнюю грань функции $f_{0} \chi_{c}$ на кубике $K_{j}$ обозначим $\gamma_{j}$, а верхнюю $-\Gamma_{j}$. Введем ступенчатые функции $\gamma$ и $\Gamma$, сужения которых на кубик $K_{j}$ равны соответственно $\gamma_{j}$ и $\Gamma_{j}$. По построению $\gamma \leqslant f_{0} \chi_{c} \leqslant \Gamma$. Далее воспользуемся соотношением

$$
I_{c, \tau}=\frac{1}{\tau} \sum_{j \in J_{c, 2}} \int_{R_{j, \tau, c}} f_{0}(a+t \omega) d t
$$

и учтем, что $\mu\left(R_{j, \tau, c}\right)=\mu\left(R_{j, \tau}\right)$, если $K_{j} \subset K_{c}$, и $\mu\left(R_{j, \tau, c}\right) \leqslant \mu\left(R_{j, \tau}\right)$ для любого $j$. Следовательно, из (3) получим двусторонние оценки

$$
\sum_{j \in J_{c, 1}} \gamma_{j} \frac{\mu\left(R_{j, \tau}\right)}{\tau} \leqslant I_{c, \tau} \leqslant \sum_{j \in J_{c, 2}} \Gamma_{j} \frac{\mu\left(R_{j, \tau}\right)}{\tau} .
$$

По следствию из теоремы усреднения [1; следствие 2, с. 252] имеем

$$
\lim _{\tau \rightarrow \infty} \frac{\mu\left(R_{j, \tau}\right)}{\tau}=\frac{\lambda\left(K_{j}\right)}{(2 \pi)^{n}} .
$$

Выберем $\tau_{1}$ так, чтобы при $\tau \geqslant \tau_{1}$ и любом $j \in J$ выполнялись неравенства

$$
\frac{\lambda\left(K_{j}\right)}{(2 \pi)^{n}}-\frac{\varepsilon}{r|J|} \leqslant \frac{\mu\left(R_{j, \tau}\right)}{\tau} \leqslant \frac{\lambda\left(K_{j}\right)}{(2 \pi)^{n}}+\frac{\varepsilon}{r|J|} .
$$


Из (4), (5) при $\tau \geqslant \tau_{1}$ и любом $c \geqslant 0$ имеем

$$
\frac{1}{(2 \pi)^{n}} \int_{K_{c, 1}} \gamma(y) d y-\varepsilon \leqslant I_{c, \tau} \leqslant \frac{1}{(2 \pi)^{n}} \int_{K_{c, 2}} \Gamma(y) d y+\varepsilon .
$$

На основании равномерной измеримости семейства множеств $\left\{K_{c}\right\}$ и определения интеграла Римана отсюда следует, что при достаточно большом $l$ и любых $\tau \geqslant \tau_{1}, c \geqslant 0$ выполняются двусторонние оценки

$$
\frac{I_{c}}{(2 \pi)^{n}}-2 \varepsilon \leqslant I_{c, \tau} \leqslant \frac{I_{c}}{(2 \pi)^{n}}+2 \varepsilon .
$$

Подобным же образом получаются оценки для меры $\mu_{c, \tau}$ :

$$
\frac{\lambda_{c}}{(2 \pi)^{n}}-2 \varepsilon \leqslant \mu_{c, \tau} \leqslant \frac{\lambda_{c}}{(2 \pi)^{n}}+2 \varepsilon
$$

которые выполняются при достаточно большом $l$ и любых $\tau \geqslant \tau_{1}, c \geqslant 0$. Полученные соотношения означают, что равномерно по $c \geqslant 0$ выполняются предельные равенства

$$
\lim _{\tau \rightarrow \infty} \mu_{c, \tau}=\frac{\lambda_{c}}{(2 \pi)^{n}}, \quad \lim _{\tau \rightarrow \infty} I_{c, \tau}=\frac{I_{c}}{(2 \pi)^{n}} .
$$

Следовательно, сначала можно выполнить предельный переход при $\tau \rightarrow \infty$ в равенстве (2) с учетом (6), а затем перейти к вычислению точной верхней границы. В результате получим

$$
M\left(f_{0}\right)=\sup _{c \geqslant 0} \varphi(k, c) .
$$

Так как $M(f)=M\left(f_{0}\right)+m(f)$, теорема доказана.

Отметим, что вычисление пределов максимальных средних связано с построением усредненных дифференциальных включений в задачах с быстрыми и медленными переменными. Если воспользоваться техникой опорных функций многозначных отображений, то усреднение опорных функций по быстрым переменным приводит к вычислениям подобного вида.

\section{СПИСОК ЦИТИРОВАННОЙ ЛИТЕРАТУРЫ}

[1] В. В. Арнольд, Математические методы классической механики, Наука, М., 1989. [2] О. П. Филатов, Матем. заметки, 59:5 (1996), 759-767.

\section{О. П. Филатов}

E-mail: filatov_oleg@samaradom.ru 